انتاج اوكسيد الخارصين الدوائي من مخلفات الخارصين

د.جهاد عبل طعبس *.

2008/1/29 تاريخ قبول النشر

الخلاصة

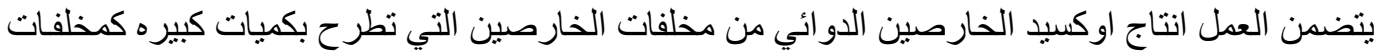

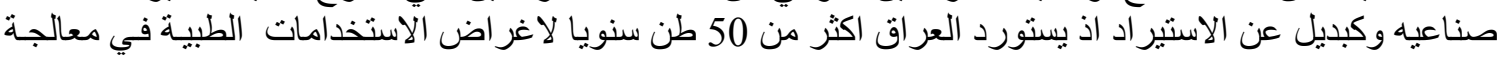

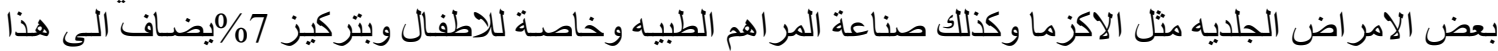

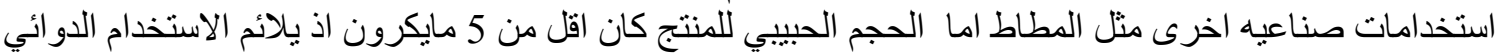

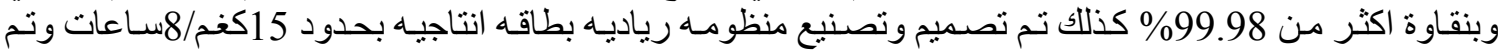
تسويق النموذج يزن 385 كغم الى ادوية سامر اءو واجتاز الاختبار بنجاح .

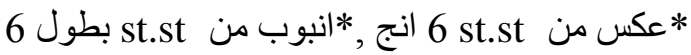
م وقطر 6 انج ,*انبوب من انج مt.st بطول 6 من موقطر 4

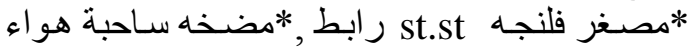

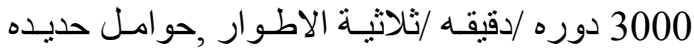
عدد 6, منصده معدنيه عدد 2 و سـايكلون بطول 1.5

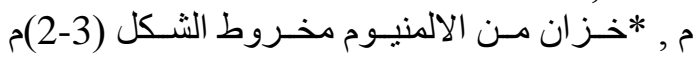

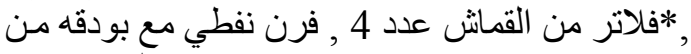

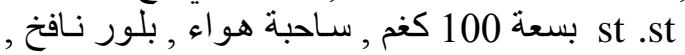

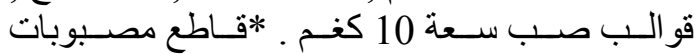
الخارصين /اوكسجين - استلين ,*قاعه مسقفه بطول 50 م وارتفاع 5 م ذات تهوين - تهويه.

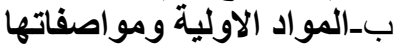

كما مبين في جدول رقم (1) Table-1-The analysis of the impurities of scrap batteries and slag before purification .

\begin{tabular}{|c|c|c|c|c|c|}
\hline Material & $\mathrm{Fe} \%$ & $\mathrm{~Pb} \%$ & $\mathrm{Cd} \%$ & $\mathrm{Zn} \%$ & Clay\% \\
\hline Scrap batteries & 1.5 & 1.0 & 0.018 & 97 & 0.1 \\
\hline Slag & 4.7 & 3.2 & 0.022 & 65 & 25 \\
\hline
\end{tabular}

\section{3. المراحل العملية :}

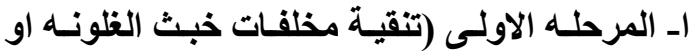

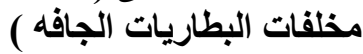

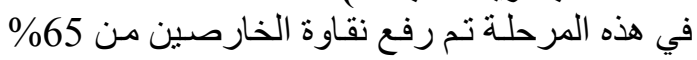

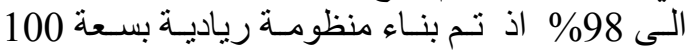

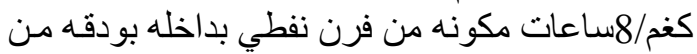

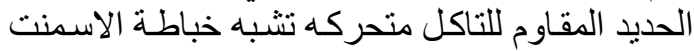

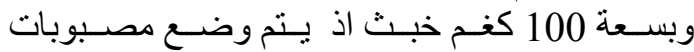

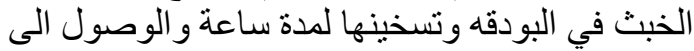

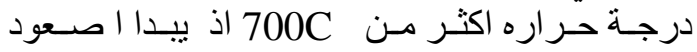
الابخرة البيض المتمثلة بالاكاسيد ويتم التخلص منها

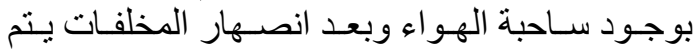

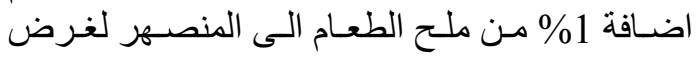

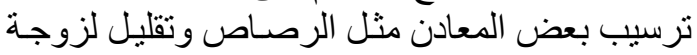
المنصهر ثم نبدا بعملية الصب في قون الرئ الب من الحديد

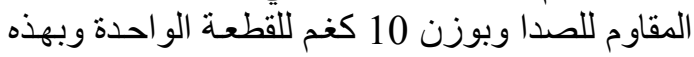

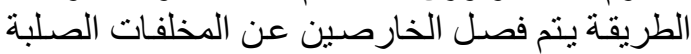
التي تبقى في اسفل البودقة وتز ال هذه المخلفات

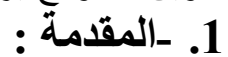

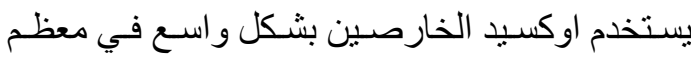
الصناعات الكبمياوية كما بيتخدم بشكل كبير جئ في في في في

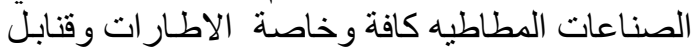

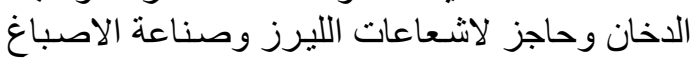

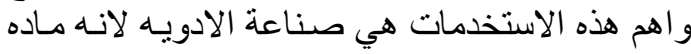

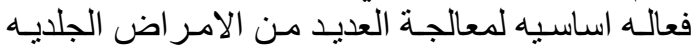

وصناعة المر اهم الطبيه (6-1-1).

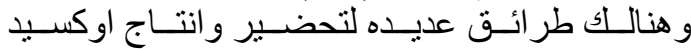

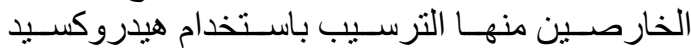
الصوديو لملح الخارصين المذاب وطريقة كاربونات الترونات

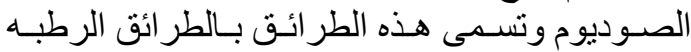
ويمكن الوصول عند استخدامها الى نقاوه \%

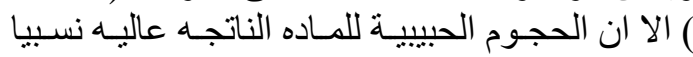

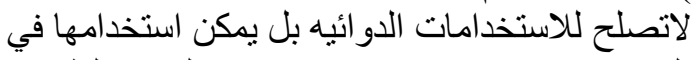

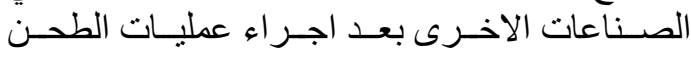

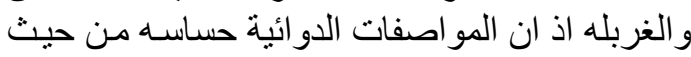

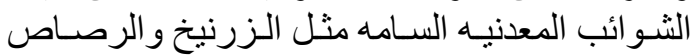

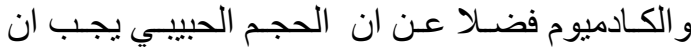

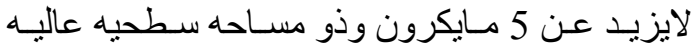

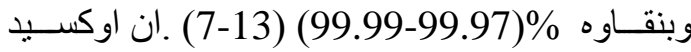

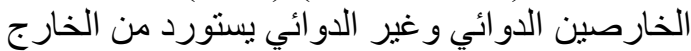

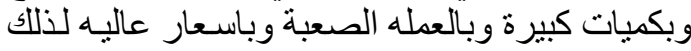

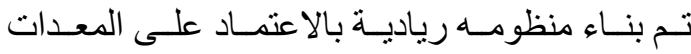

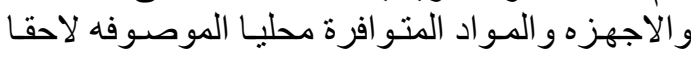

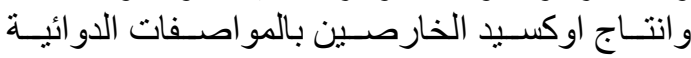
المطلوبة .

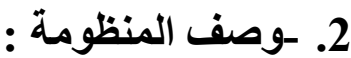
ا-_المعدات والاجهزه المستخدمه :

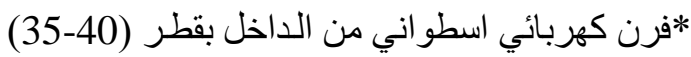

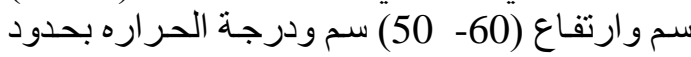

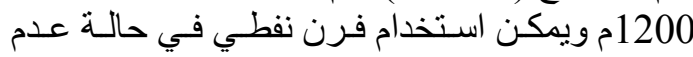

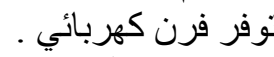
*بو دقه من الكر افييت انكليزي او الماني المنشا بسعه . 50 *انبوب مـن الكـاربون ستيل قطر ( 60-50)سم وطول( 2.52)سم وسمك 1سم . 
عن ذللك فـان بودقة التفاعل يجب ان تكون مقاومـة

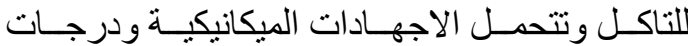

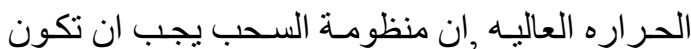

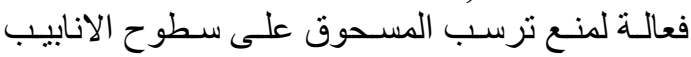

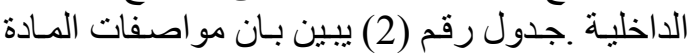

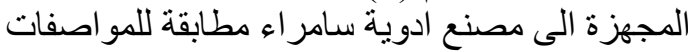

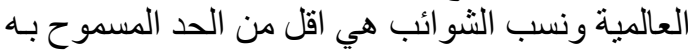

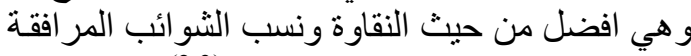
من المنتج ضمن المو اصفة العالميـة (2,3) اذ ان نسبة النية

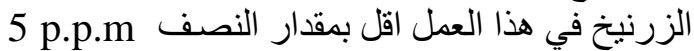

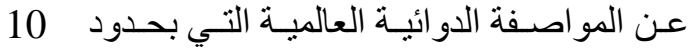

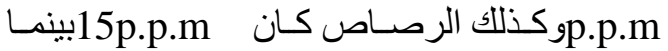

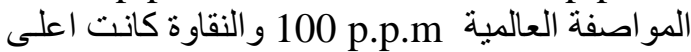

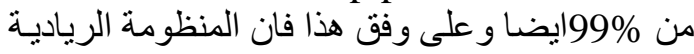

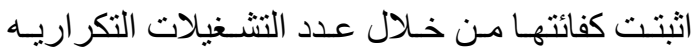

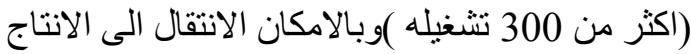
الصناعي لوضوح المسلك التكنولوجي .

Table-2-The chemical analysis report carried out at samarra plant /quality assurance department /quality control labs (Raw materials)

\begin{tabular}{|c|c|c|}
\hline Name of material & Zinc oxide in this work & Batch NO1 \\
\hline Raceived form & Accepted or Refused & Control No 2201 \\
\hline & Accepted & Specification ${ }^{(2,3)}$ \\
\hline Packing & $\begin{array}{c}15 * 25 \mathrm{~kg}+10 \mathrm{~kg} * 1 \mathrm{~B} \\
=385 \mathrm{~kg}\end{array}$ & B.p 80 \\
\hline Description & $\begin{array}{c}\text { Awhite soft powder, } \\
\text { free from grittiness odourless }\end{array}$ & \\
\hline Solubility & Accepted & \\
\hline $\begin{array}{l}\text { Loss on ignition at } \\
\mathbf{5 0 0 C}\end{array}$ & $1 \%$ & N.M.T $1 \%$ \\
\hline Acidity or Alkalinity & Accepted & Accepted \\
\hline Lead & 15 p.p.m & 100 P.P.M \\
\hline Arsenic & $\frac{p+p}{5}$ & 10 p.p.m \\
\hline Identitiy & Comply (A) & \\
\hline Assay $\mathrm{ZnO}$ & $100.2 \%$ & $(99-100.5) \%$ \\
\hline Final result & & Accepted \\
\hline
\end{tabular}

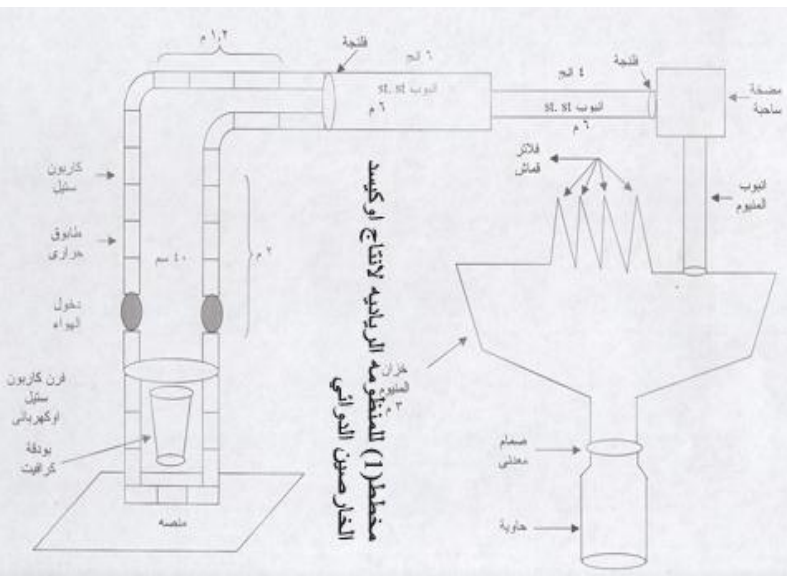

مخطط (1) المنظومة الريادية لاتتاج اوكسيد الخارصين الدوائي

1- Sharpe.A.G.Inorganic chemistry, 1981, LongmanGroup Limited ,Published in United States of America by Longman Inc, New Yourk , p566.

2- The Committee British Pharmaclopedia London .2000, 30 ed :229-230.

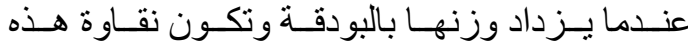
المصبوبات الناتجه بحدود \% (98-99) باليونة بـ المرحلة الثانية (انتاج اوكسيد الخارصين

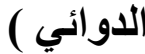
تالتالف المنظو مـة مـن فرن نفطي او كهربـائي مبطن

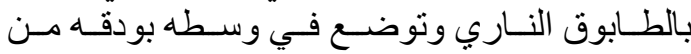

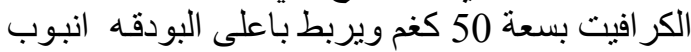

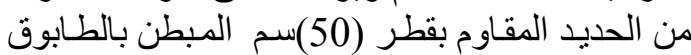

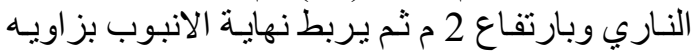

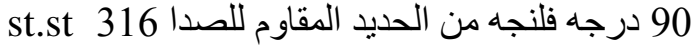

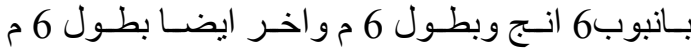

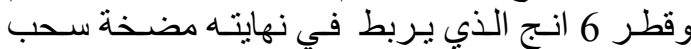

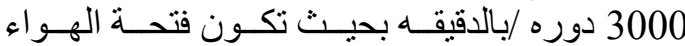

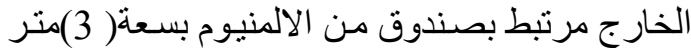

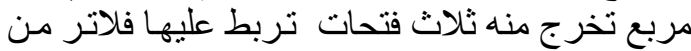

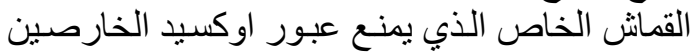

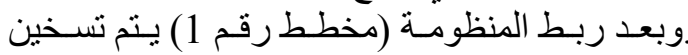

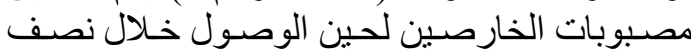

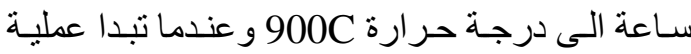
التبخير التدريجي في منصـهر الخارصين يتم تشغيل

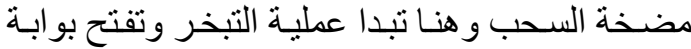

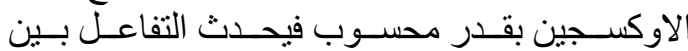

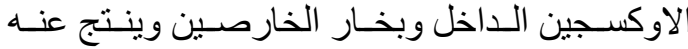

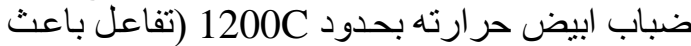

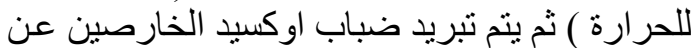

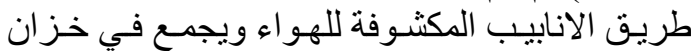

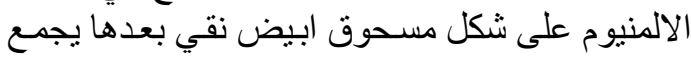
في اكياس من النايلون ويرقم ويخزن .

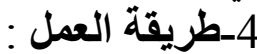
تم اجر اء العديد من التجارب و وانتاج عدة وجبـات من ون

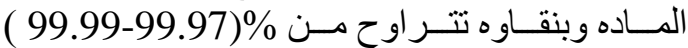

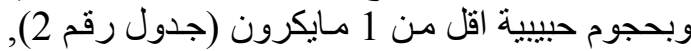

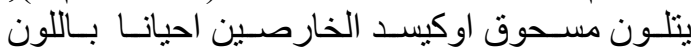

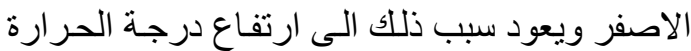

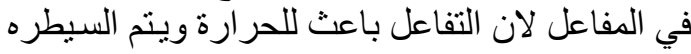

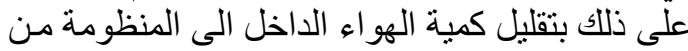

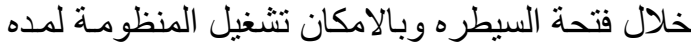

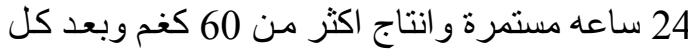

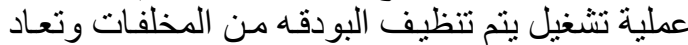

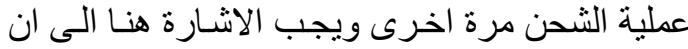

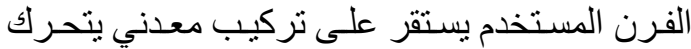

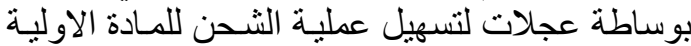

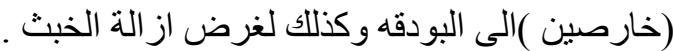

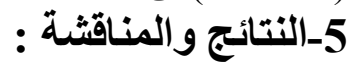

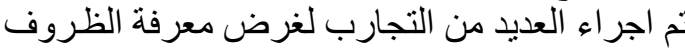

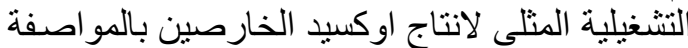

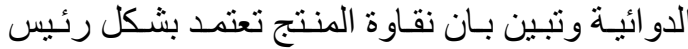

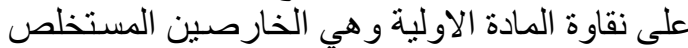

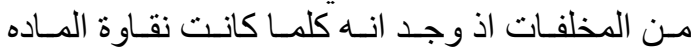

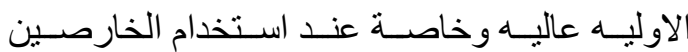

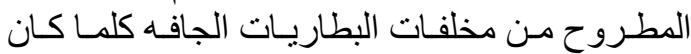

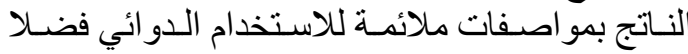


10- Soroff HS, Sasvary DH,1994. Collagenase ointment and polymixin sulfate/bacitracin spray versus silver sulfadiazine cream in partialthickness burns: a pilot study. J Burn Care \& Rehab;15:13-17.

11- Durham DR, Fortney DZ, Nanney LB.Preliminary evaluation of vibriolysin, a novel proteolytic enzyme composition suitable for the debridement of burn wound eschar. J Burn Care \& Rehab 14:520-551.

12- Monafo WW, West MA.1990,Current recommendations for topical burn therapy. Drugs;40:364373.

13- Taddonio TE, Thompson PD, Smith DJ Jr, Prasad JK,1990. A survey of wound monitoring and topical antimicrobial therapy practices in the treatment of burn injury. J Burn Care \& Rehab;11:423427.
3- Lowenson .J.D and S.Clarke,1992 Skin treatment by zinc oxide J.Biol .Chem, 267,p.5985-5995.

4- Parks.G.D.,1980,Modern Inorganic chemistry,3th,ed.,p.231-241.

5- Kneen. W.r.and p.Simpson 1982, Chemistry ,Facts,Parameter and Principle, $6^{\text {th }}$,ed p522-530.

6- Ray.H.S.1985,Extraction of Nonferrous Metals, $1^{\text {st }}$,edition ,April ,p437445.

7- Jehad .A.Taies and W.A. Mhmoud, 2002 preparation of zinc chloride for medical application,Iraqi journal of chemistry, 28(3) :631-637.

8- Jehad A.Taies and W.A.Mhamoud ,2001 preparation of zinc sulphate for medical application ,journal of science Mustansiryia,12(3):133-139.

9- Hansbrough JF, Achauer B, Dawson $\mathrm{J}$, et al 1995. Wound healing in partial-thickness burn wounds treated with collagenase ointment versus silver sulfadiazine cream. J Burn Care \& Rehab;16:241-247

\title{
Production of Zinc oxide for medical application from Zinc scraps
}

\author{
Dr.Jehad.A.Taies*
}

*Chemistry deparment/ Education College/University of Anbar

\begin{abstract}
In this work ,medical zinc oxide was produced from zinc scraps instead of traditional method which used for medical applications such as skin diseases, Iraq is importing around 50 ton/year for samarra plant the producted powder has apartical size less than 5 micron and the purity was more than $99.98 \%$,also apilot plant of yield capacitiy $15 \mathrm{~kg} / 8$ hours wsa designed and manufactured .
\end{abstract}

